\title{
Low-risk population among patients with tumor-node-metastasis stage III/IV oral squamous cell carcinoma
}

\author{
TOHRU IKEDA ${ }^{1}$, SACHIKO SEKI $^{2}$, MUTSUNORI FUJIWARA $^{3}$, MASAAKI MATSUURA $^{4}$, \\ YUU OZAKI-HONDA ${ }^{5}$, SHUICHI FUJITA ${ }^{2}$, HISAZUMI IKEDA ${ }^{6}$, MASAHIRO UMEDA $^{7}$ and IZUMI ASAHINA ${ }^{8}$ \\ ${ }^{1}$ Department of Oral Pathology, Graduate School of Medical and Dental Sciences, Tokyo Medical and Dental \\ University, Tokyo 113-8549; ${ }^{2}$ Department of Oral Pathology and Bone Metabolism, Nagasaki University Graduate \\ School of Biomedical Sciences, Nagasaki 852-8588; ${ }^{3}$ Department of Clinical Pathology, Japanese Red Cross Medical \\ Center, Tokyo 150-8935; ${ }^{4}$ Graduate School of Public Health, Teikyo University, Tokyo 173-8605; Departments \\ of ${ }^{5}$ Clinical Physiology, ${ }^{6}$ Sasebo General Hospital, Sasebo 857-8511; ${ }^{7}$ Clinical Oral Oncology and ${ }^{8}$ Regenerative \\ Oral Surgery, Nagasaki University Graduate School of Biomedical Sciences, Nagasaki 852-8588, Japan
}

Received March 7, 2016; Accepted April 16, 2017

DOI:10.3892/ol.2017.6575

\begin{abstract}
A novel system auxiliary to the Union for International Cancer Control classification may allow the prognosis of patients with malignant tumors at similar stages to be predicted, as currently this is challenging. The present study generated a novel system to predict populations at low risk among patients with stage III/IV oral squamous cell carcinoma (OSCC). A total of 41 patients who were diagnosed at stages III/IV OSCC and underwent surgical tumor resection were analyzed. Band-like or follicular lymphocyte infiltration, intraepithelial micro-abscess formation and natural killer (NK) cell infiltration were histopathologically evaluated. Cox's proportional hazards regression model was used to identify prognostic factors, and a set of factors was selected from a combination of those prognostic factors to create a logic covariate model. A logic regression analysis for 41 patients with OSCC revealed that the presence of intraepithelial micro-abscesses and a lower density of NK cells were significantly associated with a favorable prognosis among patients with stage III/IV OSCC. These results suggested that the host innate immune responses, including neutrophil and NK cell infiltrations, are
\end{abstract}

Correspondence to: Dr Tohru Ikeda, Department of Oral Pathology, Graduate School of Medical and Dental Sciences, Tokyo Medical and Dental University, 1-5-45 Yushima, Tokyo 113-8549, Japan

E-mail: tohrupth.mpa@tmd.ac.jp

Abbreviations: HE, hematoxylin and eosin; IPF, important prognostic factor; NK, natural killer; OSCC, oral squamous cell carcinoma; TNM, tumor-node-metastasis; UICC, Union for International Cancer Control; VEGF, vascular endothelial growth factor

Key words: oral squamous cell carcinoma, natural killer cell, inflammation, prognosis, multivariate analysis useful for prognostic prediction in patients with advanced malignant tumors.

\section{Introduction}

The tumor-node-metastasis (TNM) classification that was established by the Union for International Cancer Control is universally used to reliably predict the prognosis of patients with malignant tumors, and is based on the anatomical distribution of malignant tumors (1). Typically, tumors at relatively early stages (stage I/II) are distributed in a limited area and should have a better prognosis, compared with those at more advanced stages (III/IV). However, certain patients with early-stage tumors may have a poor prognosis, whereas others with advanced tumors may have a good prognosis. The aim of the present study was to create models for predicting the prognosis of malignant tumors in a manner that may be auxiliary to TNM staging.

The present study investigated the prognostic predictors of oral squamous cell carcinoma (OSCC) based on information about proteins expressed in OSCC cells combined with patients' clinical information. Vascular endothelial growth factors (VEGFs) are associated with tumor angiogenesis or lymphovasculogenesis (2-4), and a number of previous studies of oral pathology have attempted to use intracellular levels of VEGF expression in OSCC cells to predict the prognosis of patients with OSCC; however, these results were variable (5-12). In a previous study, a novel model was proposed to predict the prognosis of patients with OSCC using their age and the expression levels of VEGF-A and VEGF-C (13). It has also been revealed that podoplanin, a platelet-aggregation glycoprotein $(14,15)$ that is expressed by OSCC cells $(16-20)$, may serve as a prognostic predictor of OSCC (21). Thus the present study analyzed molecules expressed by OSCC tumor cells at all stages.

The present study also aimed to identify low-risk populations among patients with advanced OSCC (stage III/IV) using logic regression analysis, as previously described (13). Proteins 
that are expressed by tumor cells and the host responses raised against these cells can influence the prognosis of malignant tumors (22). Immunoreactions are important host responses against tumor invasion, and inflammatory cell infiltration is a key histological factor for assessing host immunoreactions against tumor infiltration (23). Variable mucosal inflammation frequently occurs in the oral cavity and is visible in a number of histopathological tissue samples (24). Considering the variability of inflammatory lesions in the oral mucosa, the prognosis of OSCC with or without inflammatory reactions may be challenging to evaluate. However, it may have specific value for separately detecting innate and adaptive immunity if included in the logic regression analysis. The present study analyzed the ability of neutrophils and natural killer (NK) cells to detect innate immunity, and of lymphocytes to detect adaptive immunity, and then applied these to a statistical model to predict low-risk populations in patients with stage III/IV OSCC.

\section{Materials and methods}

Patients and materials. The present study analyzed data from 41 patients (male, $n=30$; female, $n=11$; agerange, $31-85$-years-old) who were admitted to Nagasaki University Dental Hospital (Nagasaki, Japan) between October 1986 and September 2002, with histopathologically diagnosed stage III/IV OSCC and underwent surgical tumor resection. Histopathological analysis diagnosed 31 patients with well-differentiated OSCC and 5 each with moderately- and poorly-differentiated OSCC, respectively. A total of 22 patients were treated with preoperative irradiation (total, $30 \mathrm{~Gy}$ ) using a Clinac $2100 \mathrm{C}$ linear accelerator (Varian Medical Systems, Palo Alto, California, USA) and pepleomycin. Of the 41 aforementioned patients, 4 patients were treated with preoperative irradiation only, 8 patients were treated with preoperative pepleomycin only and 7 patients were not treated prior to surgery. All patients were followed up at the hospital until 2005; overall survival was analyzed, and 34 patients succumbed to disease and 7 survived during the follow-up period. The present study was approved by the Ethics Committee of Nagasaki University Hospital (Nagasaki, Japan; approval no. 16020839).

Histopathological analyses. Tissue sections (3- $\mu \mathrm{m}$ thick) were cut from paraffin-embedded blocks of biopsy or surgical tissue samples. Sections from patients with OSCC were stained with hematoxylin and eosin (HE) using a standard protocol. After deparaffinization, sections were stained with Meyer's hematoxylin solution at room temperature for $5 \mathrm{~min}$. After rinsing under running water for $15 \mathrm{~min}$, sections were further stained with eosin solution for $3 \mathrm{~min}$ at room temperature. Lymphocyte infiltration was then graded by light microscopy (magnification, x40) as follows: 1, tissue samples with band-like or follicular lymphocyte infiltration at $\mathrm{x} 40$ magnification were categorized as having dense infiltration; 0, no band-like or follicular lymphocyte infiltration. Neutrophil infiltration was evaluated as follows: 1, presence or 0 , absence of intraepithelial micro-abscesses of OSCC in HE-stained tissue samples. Tumors were also assessed in HE-stained sections as well, moderately or poorly differentiated, which were categorized as 0,1 and 2, respectively. The mode of invasion was categorized as 0,1 or 2 , which representing Jakobsson's grade $1 / 2,3$ and 4 , respectively (25). NK cells were stained using an antibody against NKp46 (dilution, 1:50; cat. no. MAB1850; R\&D Systems, Inc., Minneapolis, MN, USA), as previously described (13) and samples were graded as -, 1 or 2 using light microscopy, indicating sample staining was undetectable, in $<5$ or $\geq 5$ cells, respectively, in each whole specimen. In brief, deparaffinized sections were treated with $2 \%$ bovine serum albumin diluted with PBS at room temperature for $30 \mathrm{~min}$, prior to incubation with antibody overnight at $4^{\circ} \mathrm{C}$. Immunohistochemical detection was carried out using the EnVision+ system (Dako; Agilent Technologies, Inc., Santa Clara, CA, USA). Specimens were analyzed by light microscopy.

Statistical analysis. Prognostic status was defined for patients who succumbed during the observation period as poor and for patients who survived as good. The overall survival time was defined as the duration from the date of the initial surgery and the date of mortality from any cause, or the date of last contact during the follow-up period for survivors. To identify the prognostic factors, Cox's proportional hazards regression model was used for univariate and multivariate analyses, as previously reported (13). Factors were selected from a combination of prognostic factors to create a logic covariate model, and Akaike's Information Criterion (AIC) was used for the model selection (26). Log-rank test with $\chi^{2}$ statistics was used to test survival curves. Statistical procedures were performed using Statistical Language R-3.2.5 (27). $\mathrm{P}<0.05$ was considered to indicate a statistically significant difference.

\section{Results}

Histopathological analyses. Lymphocytes and neutrophils were detected in all analyzed tissue samples. Lymphocytes formed band-like or follicular infiltrates in tissue samples with dense infiltration (Fig. 1A). However, few tissue samples exhibited intraepithelial micro-abscesses (Fig. 1B). Table I summarizes the histopathological findings. NKp46-positive cells were detected in 14 of the 41 tissue samples (Table I). Cells positive for NKp46 were sparse even in tissue samples categorized as 2 (Fig. 1D). NKp46-potitive cells were absent in 27 of the 41 tissue samples (Table I). NKp46-positive cells were identified in the stromal regions of biopsy and surgical tissue samples; the positive cells were not always contact with tumor cells (Fig. 1C and D).

Statistical findings. Univariate analysis of sex, age, clinical stage, T-stage, N-stage, dense lymphocyte infiltration, microabscess, differentiation grade, mode of invasion and NKp46 expression revealed that dense lymphocyte infiltration and the presence of intraepithelial micro-abscesses predicted the good prognosis of patients with OSCC, whereas NKp64 alone was not significantly associated with a good prognosis (Table I). Logic combinations of covariates used in the univariate analysis, including dense lymphocyte infiltration, micro-abscesses, NKp46 and patients' age were further analyzed using the Cox's proportional hazards model. It was revealed that logic combinations of NKp46 and micro-abscesses had a smaller P-value (0.001) and a smaller AIC (201.8), compared with the other univariate and multivariate models. Logic combinations that 
Table I. Study subjects by factors and their estimated RR.

\begin{tabular}{|c|c|c|c|c|c|c|}
\hline \multirow[b]{2}{*}{ Factor } & \multirow[b]{2}{*}{ Category } & \multirow{2}{*}{$\begin{array}{l}\text { Sample } \\
\text { size }\end{array}$} & \multicolumn{2}{|c|}{ Prognosis } & \multicolumn{2}{|c|}{ Cox Regression } \\
\hline & & & Good & Poor & $\mathrm{RR}$ & P-value \\
\hline \multirow[t]{2}{*}{ Sex } & 0 & 11 & 1 & 10 & 1.000 & - \\
\hline & 1 & 30 & 6 & 24 & 0.733 & 0.416 \\
\hline \multirow[t]{2}{*}{ Age } & -69 & 22 & 5 & 17 & 1.000 & - \\
\hline & $70-$ & 19 & 2 & 17 & 1.577 & 0.187 \\
\hline \multirow[t]{2}{*}{ Stage } & III & 9 & 2 & 7 & 1.000 & - \\
\hline & IV & 32 & 5 & 27 & 1.475 & 0.362 \\
\hline \multirow[t]{3}{*}{ T-stage } & 1,2 & 13 & 3 & 10 & 1.000 & - \\
\hline & 3 & 4 & 1 & 3 & 1.012 & 0.985 \\
\hline & 4 & 24 & 3 & 21 & 1.689 & 0.176 \\
\hline \multirow[t]{3}{*}{ N-stage } & 0 & 6 & 1 & 5 & 1.000 & - \\
\hline & 1 & 13 & 2 & 11 & 0.777 & 0.640 \\
\hline & 2 & 22 & 4 & 18 & 0.761 & 0.590 \\
\hline \multirow[t]{2}{*}{ Dense lymphocyte infiltration } & 0 & 24 & 1 & 23 & 1.000 & - \\
\hline & 1 & 17 & 6 & 11 & 0.471 & 0.044 \\
\hline \multirow[t]{2}{*}{ Microabscess } & 0 & 33 & 2 & 31 & 1.000 & - \\
\hline & 1 & 8 & 5 & 3 & 0.221 & 0.014 \\
\hline \multirow[t]{3}{*}{ Differentiation grade } & 0 & 5 & 2 & 3 & 1.000 & - \\
\hline & 1 & 5 & 1 & 4 & 1.770 & 0.458 \\
\hline & 2 & 31 & 4 & 27 & 1.571 & 0.460 \\
\hline \multirow[t]{2}{*}{ Mode of invasion } & 0 & 20 & 2 & 18 & 1.000 & - \\
\hline & 1,2 & 21 & 5 & 16 & 0.732 & 0.368 \\
\hline \multirow[t]{3}{*}{ NKp46 } & 0 & 27 & 6 & 21 & 1.000 & - \\
\hline & 1 & 10 & 1 & 9 & 1.222 & 0.619 \\
\hline & 2 & 4 & 0 & 4 & 1.496 & 0.473 \\
\hline
\end{tabular}

RR, relative risk; T, tumor; $\mathrm{N}$, node.
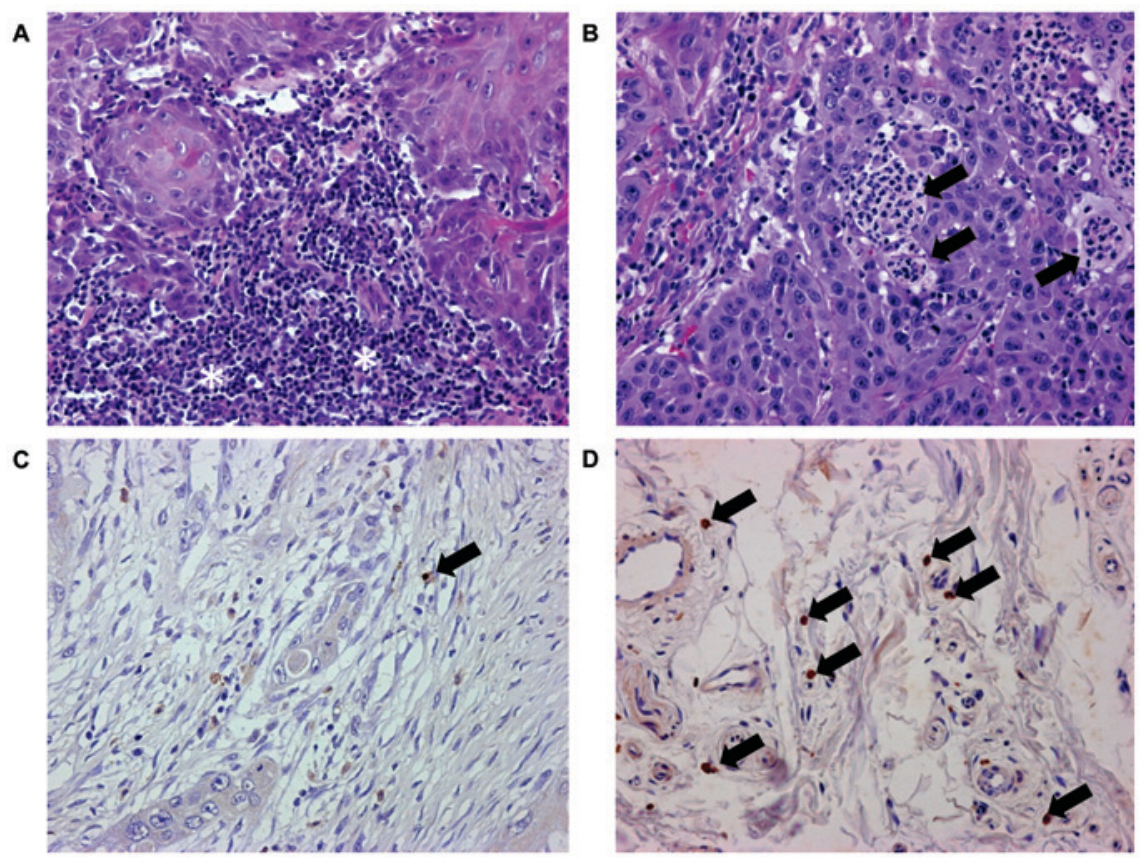

Figure 1. Representative images of OSCC tissue samples with dense lymphocyte infiltration and micro-abscesses. (A) *Dense lymphocyte infiltration. (B) Arrows indicate micro-abscesses. Representative expression profiles of NKp46 categorized as (C) 1 and (D) 2. The arrows in C and D indicate NKp46-positive cells. Original magnification, x200. OSCC, oral squamous cell carcinoma; NK, natural killer. 
Table II. Results of Cox proportional hazards regression models (N=41).

\begin{tabular}{|c|c|c|c|c|c|c|c|}
\hline \multirow[b]{2}{*}{ Covariate } & \multirow[b]{2}{*}{ Estimated coefficients } & \multirow[b]{2}{*}{ SE } & \multirow[b]{2}{*}{ P-value } & \multicolumn{3}{|c|}{$\begin{array}{l}95 \% \text { confidence } \\
\text { interval }\end{array}$} & \multirow[b]{2}{*}{ AIC } \\
\hline & & & & $\mathrm{RR}$ & Lower & Upper & \\
\hline \multicolumn{8}{|l|}{ Selected logic regression model } \\
\hline $\mathrm{IPF}^{\mathrm{a}}$ & -1.875 & 0.74 & 0.11 & 0.15 & 0.04 & 0.65 & 201.8 \\
\hline \multicolumn{8}{|c|}{$\begin{array}{l}\text { Other univariate and multivariate models } \\
\text { with a linear combination of covariates }\end{array}$} \\
\hline \multicolumn{8}{|l|}{ Univariate model 1} \\
\hline Dense lymphocyte infiltration & -0.752 & 0.37 & 0.044 & 0.47 & 0.23 & 0.98 & 208.4 \\
\hline \multicolumn{8}{|l|}{ Univariate model 2} \\
\hline Microabscess & -1.510 & 0.61 & 0.014 & 0.22 & 0.07 & 0.73 & 203.8 \\
\hline \multicolumn{8}{|l|}{ Multivariate model } \\
\hline Dense lymphocyte infiltration & -0.170 & 0.41 & 0.681 & 0.84 & 0.38 & 1.90 & 205.6 \\
\hline Microabscess & -1.390 & 0.68 & 0.041 & 0.25 & 0.07 & 0.95 & \\
\hline
\end{tabular}

${ }^{\mathrm{a}} \mathrm{N}$; number of patients in the model. IPF $=1$ when $\mathrm{NKp} 46=(0$ or 1$)$ and microabscess $=1, \mathrm{IPF}=0$ when $(\mathrm{NKp} 46=2)$ or $(\mathrm{NKp} 46=(0$ or 1$)$ and microabscess=0). SE, standard error; AIC, Akaike's Information Criterion; IPF; important prognostic factors.

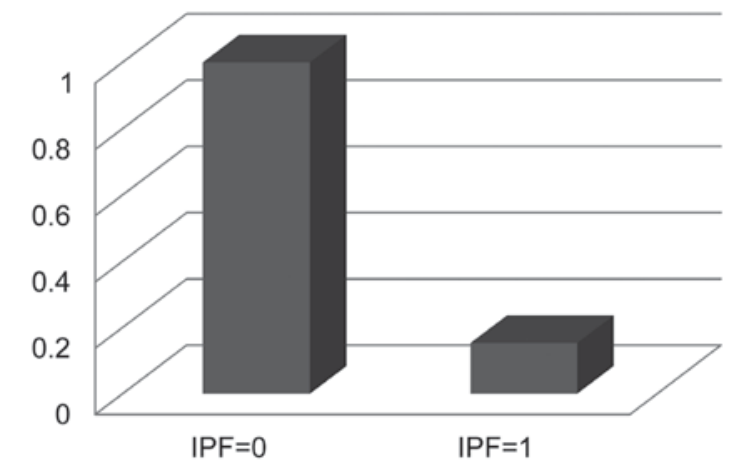

Figure 2. Estimated relative risk for patients with stage III/IV OSCC as evaluated by IPF. IPF $=1$ when $\mathrm{NKp} 46=0$, or IPF $=1$ and micro-abscesses $=1$. IPF $=0$ when $\mathrm{NKp} 46=2$, or NKp46=0 or 1 and micro-abscesses $=0$. IPF, important prognostic factor; OSCC, oral squamous cell carcinoma; NK, natural killer.

were significantly associated with the lowest P-value (0.001) in patients with advanced OSCC were named important prognostic factors (IPF). An IPF had a value of 1 when NKp46 expression $<2$ (when the number of positive cells $<5$ at $\mathrm{x} 100$ magnification in each specimen) and micro-abscesses $=1$ (presence of intraepithelial micro-abscesses of OSCC), and a value of 0 otherwise (Table II). Fig. 2 presents the estimated relative risk for stage III/IV OSCC evaluated by IPF, and Fig. 3 depicts the estimated survival curves for patients with stage III/IV OSCC and each IPF status. Patients with an IPF of 1 had statistically significant favorable survival as calculated using a Log-rank test $(\mathrm{P}=0.004)$.

\section{Discussion}

In a cohort of patients with OSCC, the host defense system was analyzed based on inflammatory reactions and NK cell infiltration; subsequently, a novel logic combination model

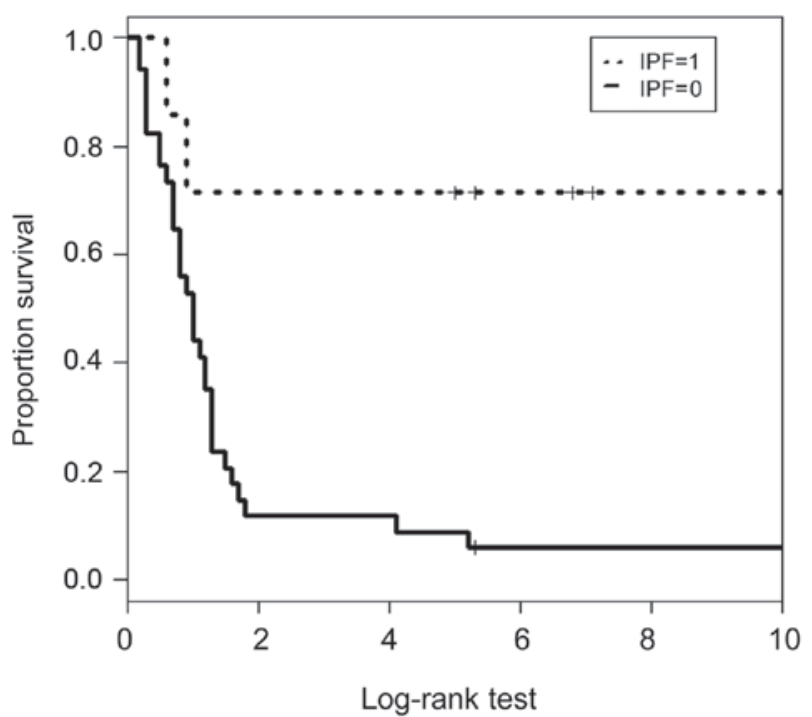

$\begin{array}{lr}\text { Chi-square } & 8.53 \\ \text { P-value } & 0.004\end{array}$

Figure 3. Kaplan-Meier estimator curves for patients with stage III/IV OSCC evaluated by IPF. IPF, important prognostic factor, IPF $=1$ when NKp46=(0 or 1$)$ and Microabscess $=1, \mathrm{IPF}=0$ when $(\mathrm{NKp} 46=2)$ or $(\mathrm{NKp} 46=(0$ or 1$)$ and Microabscess $=0$ ); OSCC, oral squamous cell carcinoma. The $\mathrm{x}$-axis is the observation period (year) and the $y$-axis is the survival rate.

was proposed using intraepithelial micro-abscess formation and NK cell infiltration as covariates to identify low risk populations in patients with advanced OSCC. In a previous study from our group, a logic combination model was proposed for predicting the prognosis of patients OSCC using the expression levels of VEGF-A and VEGF-C: This combination model appeared to predict the prognosis of patients with stage I and II OSCC (12). Therefore, the previous study (12) hypothesized 
that proteins expressed by tumor cells that correlate with tumor malignancy may predict the poor prognosis of patients with early-stage OSCC. The present study proposed a logic combination model using host responses as covariates to predict the good prognosis of patients with stage III/IV OSCC. Host responses, including inflammatory reactions may also have been more applicable in predicting the prognosis of patients with advanced, rather than early-stage, disease when the host responses against tumors were evident.

Lymphocytes use numerous mechanisms to eliminate tumor cells, including direct attack by cytotoxic lymphocytes (23). Infiltration by lymphocytes may be associated with the prognosis of patients with malignant tumors, including OSCC (28). In the present study, univariate analysis revealed an association between dense lymphocyte infiltration and an improved prognosis. The present study immunohistochemically distinguished other lymphocyte populations from NK cells, which attack tumor cells without recognizing major histocompatibility complex class I molecules and are part of the innate immune response (23). In the present study, NK cells were sparse in the biopsy and surgical tissue samples, as previously reported $(29,30)$, despite reports of dense infiltration of NK cells in OSCC (31). Therefore, NK cells may have a limited ability to eliminate OSCC cells at advanced stages. In contrast to dense lymphocyte infiltration, univariate analysis did not reveal an association between NK cell density and the prognosis of patients with OSCC. The present study also analyzed host response based on the presence of intraepithelial micro-abscesses. Direct evidence of neutrophil contribution to protection against tumors has not previously been reported. In this study, univariate analysis has demonstrated a close associated of neutrophils with prognosis of OSCC. Thus, the present study assumed that intraepithelial micro-abscesses do not directly eliminate tumor cells, but reflect the immune competence of patients with OSCC to a certain extent.

Variables revealed to be significantly associated by univariate analysis usually serve as covariates in further analyses. Lymphocyte infiltration and micro-abscess formation were significantly associated with the prognosis of patients with OSCC in univariate analysis, and the present study also further analyzed NK cell density. The logic combination model named IPF, in which patients with OSCC exhibited intraepithelial micro-abscesses and a lower density of NK cells, revealed the lowest AIC scores and a predicted 6-fold lower risk of a poor prognosis, compared with other patients. Furthermore, considering the biological function of NK cells in tumor immunity, patients with a higher density of NK cells experienced a markedly higher risk of having a poor prognosis. The biological explanation may suggest that NK cells tend to appear in response to highly malignant tumor cells at advanced stages.

The biological mechanisms underlying inflammation-associated carcinogenesis have been investigated in association with chemokines $(32,33)$. Crosstalk via chemokines and their receptors among tumor cells, tumor stromal cells and endothelial cells of tumor-associated blood vessels contribute to the progression of tumors in vivo (34). However, the inhibition of the chemokine $\mathrm{C}-\mathrm{C}$ motif chemokine ligand 2 accelerated breast cancer metastasis in animal models; therefore, understanding of the roles of chemokines on carcinogenesis and tumor progression must be improved (22). In addition, lymphocytes may promote tumor metastasis in association with their affinity for endothelial cells by forming tumor cell-lymphocyte chimeras (35). These hypotheses do not comply with the notion of tumor immunity, which is an advantageous biological reaction to remove dangerous transformed tumor cells, and indicates a biphasic biological role in the inflammatory reaction to tumor cells. The biphasic role may be associated with a relatively limited contribution of lymphocyte infiltration to prognostic prediction for patients with OSCC. Furthermore, oral mucosa is often accompanied by various degrees of inflammation and the prevalence of immune-mediated diseases, including lichen planus as well as allergic reactions, are high in oral mucosa (24). This may partially explain the inflammatory reaction and why the prognosis of patients with OSCC is difficult to predict.

In conclusion, the present study developed a logic combination model to predict low risk populations among patients with stage III/IV OSCC using intraepithelial micro-abscesses and NK cell density as covariates. Host responses to tumor infiltration may be effective in evaluating the prognosis of patients with advanced OSCC.

\section{Acknowledgements}

The present study was supported by the Japan Society for the Promotion of Science (grant no. 25462865).

\section{References}

1. Brierley JD, Gospodarowicz MK and Wittekind C (eds.): TNM Classification of Malignant Tumours. Wiley Blackwell, Oxford, 2017.

2. Ferrara N, Gerber HP and LeCouter J: The biology of VEGF and its receptors. Nat Med 9: 669-676, 2003.

3. Lohela M, Bry M, Tammela T and Alitalo K: VEGFs and receptors involved in angiogenesis versus lymphangiogenesis. Curr Opin Cell Biol 21: 154-165, 2009.

4. Matsuura M, Onimaru M, Yonemitsu Y, Suzuki H, Nakano T, Ishibashi $\mathrm{H}$, Shirasuna $\mathrm{K}$ and Sueishi K: Autocrine loop between vascular endothelial growth factor (VEGF)-C and VEGF receptor-3 positively regulates tumor-associated lymphangiogenesis in oral squamoid cancer cells. Am J Pathol 175: 1709-1721, 2009.

5. Maeda T, Matsumura S, Hiranuma H, Jikko A, Furukawa S, Ishida $\mathrm{T}$ and Fuchihata $\mathrm{H}$ : Expression of vascular endothelial growth factor in human oral squamous cell carcinoma: Its association with tumour progression and p53 gene status. J Clin Pathol 51: 771-775, 1998.

6. Smith BD, Smith GL, Carter D, Sasaki CT and Haffty BG: Prognostic significance of vascular endothelial growth factor protein levels in oral and oropharyngeal squamous cell carcinoma. J Clin Oncol 18: 2046-2052, 2000.

7. Shang ZJ, Li JR and Li ZB: Circulating levels of vascular endothelial growth factor in patients with oral squamous cell carcinoma. Int J Oral Maxillofac Surg 31: 495-498, 2002.

8. Johnstone S and Logan RM: The role of vascular endothelial growth factor (VEGF) in oral dysplasia and oral squamous cell carcinoma. Oral Oncol 42: 337-342, 2006.

9. Schimming R, Reusch P, Kuschnierz J and Schmelzeisen R: Angiogenic factors in squamous cell carcinoma of the oral cavity: Do they have prognostic relevance? J Craniomaxillofac Surg 32: 176-181, 2004.

10. Shintani S, Li C, Ishikawa T, Mihara M, Nakashiro K and Hamakawa $\mathrm{H}$ : Expression of vascular endothelial growth factor A, B, C, and D in oral squamous cell carcinoma. Oral Oncol 40: $13-20,2004$.

11. Sugiura T, Inoue Y, Matsuki R, Ishii K, Takahashi M, Abe M and Shirasuna K: VEGF-C and VEGF-D expression is correlated with lymphatic vessel density and lymph node metastasis in oral squamous cell carcinoma: Implications for use as a prognostic marker. Int J Oncol 34: 673-680, 2009. 
12. Suzuki H, Fukuyama R, Hasegawa Y, Tamaki T, Nishio M, Nakashima $T$ and Tatematsu M: Tumor thickness, depth of invasion, and Bcl-2 expression are correlated with FDG-uptake in oral squamous cell carcinomas. Oral Oncol 45: 891-897, 2009.

13. Seki S, Fujiwara M, Matsuura M, Fujita S, Ikeda H, Asahina I and Ikeda T: Prediction of outcome of patients with oral squamous cell carcinoma using vascular invasion and the strongly positive expression of vascular endothelial growth factors. Oral Oncol 47: 588-593, 2011.

14. Kato Y, Fujita N, Kunita A, Sato S, Kaneko M, Osawa M and Tsuruo T: Molecular identification of Aggrus/T1alpha as a platelet aggregation-inducing factor expressed in colorectal tumors. J Biol Chem 278: 51599-51605, 2003.

15. Toyoshima M, Nakajima M, Yamori T and Tsuruo T: Purification and characterization of the platelet-aggregating sialoglycoprotein gp44 expressed by highly metastatic variant cells of mouse colon adenocarcinoma 26. Cancer Res 55: 767-773, 1995.

16. Yuan P, Temam S, El-Naggar A, Zhou X, Liu DD, Lee JJ and Mao L: Overexpression of podoplanin in oral cancer and its association with poor clinical outcome. Cancer 107: 563-569, 2006.

17. Kreppel M, Drebber U, Wedemeyer I, Eich HT, Backhaus T, Zöller JE and Scheer M: Podoplanin expression predicts prognosis in patients with oral squamous cell carcinoma treated with neoadjuvant radiochemotherapy. Oral Oncol 47: 873-878, 2011.

18. Bartuli FN, Luciani F, Caddeo F, Compagni S, Piva P, Ottria L and Arcuri C: Podoplanin in the development and progression of oral cavity cancer: A preliminary study. Oral Implantol (Rome) 5: 33-41, 2012.

19. Funayama A, Cheng J, Maruyama S, Yamazaki M, Kobayashi T, Syafriadi M, Kundu S, Shingaki S, Saito C and Saku T: Enhanced expression of podoplanin in oral carcinomas in situ and squamous cell carcinomas. Pathobiology 78: 171-180, 2011.

20. Kawaguchi H, El-Naggar AK, Papadimitrakopoulou V, Ren H, Fan YH, Feng L, Lee JJ, Kim E, Hong WK, Lippman SM and Mao L: Podoplanin: A novel marker for oral cancer risk in patients with oral premalignancy. J Clin Oncol 26: 354-360, 2008.

21. Seki S, Fujiwara M, Matsuura M, Fujita S, Ikeda H, Umeda M, Asahina I and Ikeda T: Prognostic value of podoplanin expression in oral squamous cell carcinoma-a regression model auxiliary to UICC classification. Pathol Oncol Res 20: 521-528, 2014

22. Bonapace L, Coissieux MM, Wyckoff J, Mertz KD, Varga Z, Junt T and Bentires-Alj M: Cessation of CCL2 inhibition accelerates breast cancer metastasis by promoting angiogenesis. Nature 515: 130-133, 2014.
23. Abbas AK, Lichtman AH and Pillai S: Cellular and Molecular Immunology 7 th edition. Elsevier, Philadelphia, pp389-405, 2012.

24. Neville BWN, Damm DD, Allen CM and Chi AC: Oral and Maxillofacial Pathology 4th edition. 2016.

25. Jakobsson PA, Eneroth CM, Killander D, Moberger G and Mårtensson B: Histologic classification and grading of malignancy in carcinoma of the larynx. Acta Radiol Ther Phys Biol 12: $1-8,1973$

26. Akaike $\mathrm{H}$ : Information theory and an extention of the maximum likelihood principle. In: 2nd International Symposium on Information Theory. Petrov BN and Csaki F (eds.), Budapest, 1973.

27. Ihaka R and Gentleman R: R: A language for data analysis and graphics. J Comp Graph Stat 5: 299-314, 1996.

28. Chatzistamou I, Rodriguez J, Jouffroy T, Girod A, Point D, Sklavounou A, Kittas C, Sastre-Garau X and Klijanienko J: Prognostic significance of tumor shape and stromal chronic inflammatory infiltration in squamous cell carcinomas of the oral tongue. J Oral Pathol Med 39: 667-671, 2010.

29. Halama N, Braun M, Kahlert C, Spille A, Quack C, Rahbari N, Koch M, Weitz J, Kloor M, Zoernig I, et al: Natural killer cells are scarce in colorectal carcinoma tissue despite high levels of chemokines and cytokines. Clin Cancer Res 17: 678-689, 2011.

30. Turkseven MR and Oygür T: Evaluation of natural killer cell defense in oral squamous cell carcinoma. Oral Oncol 46: e34-e37, 2010.

31. Katou F, Ohtani H, Watanabe Y, Nakayama T, Yoshie O and Hashimoto K: Differing phenotypes between intraepithelial and stromal lymphocytes in early-stage tongue cancer. Cancer Res 67: 11195-11201, 2007.

32. Landskron G, De la Fuente $M$, Thuwajit $P$, Thuwajit $C$ and Hermoso MA: Chronic inflammation and cytokines in the tumor microenvironment. J Immunol Res 2014: 149185, 2014.

33. Mukaida N, Sasaki S and Baba T: Chemokines in cancer development and progression and their potential as targeting molecules for cancer treatment. Mediators Inflamm 2014: 170381, 2014

34. Qian BZ, Li J, Zhang H, Kitamura T, Zhang J, Campion LR, Kaiser EA, Snyder LA and Pollard JW: CCL2 recruits inflammatory monocytes to facilitate breast-tumour metastasis. Nature 475: 222-225, 2011.

35. Song G, Ren J, Stojadinovic A, Chen W, Sahab Z, Fu SW and Man YG: Conjunction of tumor cells with lymphocytes: Implications for tumor invasion and metastasis. Cancer Epidemiol 36: 354-363, 2012 